\title{
Mediastinal Neurofibroma Originating from the Pulmonary Branch of the Right Vagus Nerve in a Patient without von Recklinghausen Disease
}

\author{
Junichi Okamoto ${ }^{1,2}$ Hirotoshi Kubokura ${ }^{1,2}$ Hideaki Ishii $^{3}$ Jitsuo Usuda ${ }^{2}$
}

${ }^{1}$ Department of Thoracic Surgery, Nippon Medical School MusashiKosugi Hospital, Kawasaki, Tokyo, Japan

2 Department of Thoracic Surgery, Nippon Medical School, Bunkyo-ku, Tokyo, Japan

${ }^{3}$ Department of Pathology, Nippon Medical School Hospital, Bunkyoku, Tokyo, Japan
Address for correspondence Junichi Okamoto, MD, PhD, Department of Thoracic Surgery, Nippon Medical School Musashi-Kosugi Hospital, 1-396 Kosugi-cho, Nakahara-ku, Kawasaki, Kanagawa, Japan 211-8533 (e-mail: s3021@nms.ac.jp).

Thorac Cardiovasc Surg Rep 2013;2:29-31.

\begin{abstract}
Keywords

- neurofibroma

- vagus nerves

- VATS

Intrathoracic neurofibromas originating from the vagus nerve in patients without von Recklinghausen disease is rare and poses a problem in etiological diagnosis. Surgical resection is usually necessary for precise diagnosis of such tumors.

We report the first case of a neurofibroma originating from the right pulmonary branch of the vagus nerve in a 34-year-old male without von Recklinghausen disease.

The diagnosis was suggested by the radiological features and was confirmed histologically after resection.
\end{abstract}

\section{Introduction}

Neurogenic tumors of the mediastinum constitute 20 to $30 \%$ of all mediastinal tumors. ${ }^{1}$ Most neurogenic tumors are schwannomas located on the intercostal or intrathoracic phrenic nerves. ${ }^{2}$ Although a primary tumor of the intrathoracic vagus nerve is rare, a neurofibroma originating from the vagus nerve is often associated with von Recklinghausen disease. ${ }^{2,3}$ Tumors originating from the vagus nerve and not associated with von Recklinghausen disease have been reported to have an incidence rate of approximately $0.1 \% .{ }^{1-3}$ Saito et al reported that with the exception of their one case, they were unable to find any other report in the literature of a mediastinal neurofibroma originating from the phrenic nerve in patients without von Recklinghausen disease. ${ }^{1}$

We present a rare case of a neurofibroma originating from the pulmonary branch of the right vagus nerve in a patient without von Recklinghausen disease.

\section{Case Report}

A 34-year-old Japanese man was referred to our hospital for evaluation of an abnormal shadow on a chest radiograph.
He did not have any symptom of von Recklinghausen disease such as café-au-lait spots, skeletal abnormalities, hamaltomas of the iris, and other freckling. His family and medical histories were unremarkable. He and his family did not suffer from von Recklinghausen disease, although he had been smoking for 15 years. Physical examination was unremarkable, and the results of laboratory data, blood gas analysis, electrocardiography, and pulmonary function tests were normal. A chest radiograph showed a smooth, round shadow with extrapleural and hilum overlay signs in the right middle lung field (-Fig. 1a). Computed tomography revealed this shadow as a solitary mass behind the right main bronchus, with a diameter of $3.5 \mathrm{~cm}$ ( $\mathbf{- F i g . ~ 1 b )}$. T1-weighted magnetic resonance imaging (MRI) showed a lesion behind the right main bronchus with intermediate signal intensity (data not shown), whereas T2-weighted MRI revealed a high-intensity lesion with a part showing low-signal intensity (-Fig. 1c). Considering these findings, the lesion was diagnosed as a bronchogenic cyst and surgical resection was planned.

We performed video-assisted thoracic surgery (VATS) to resect the tumor with a $10-\mathrm{cm}$ skin incision. A round tumor, received

April 1, 2013

accepted after revision

May 17, 2013

published online

July 23, 2013
DOI http://dx.doi.org/

10.1055/s-0033-1348477. ISSN 2194-7635. (c) 2013 Georg Thieme Verlag KG
Stuttgart · New York

License terms

(1) $\Theta \circledast$ 

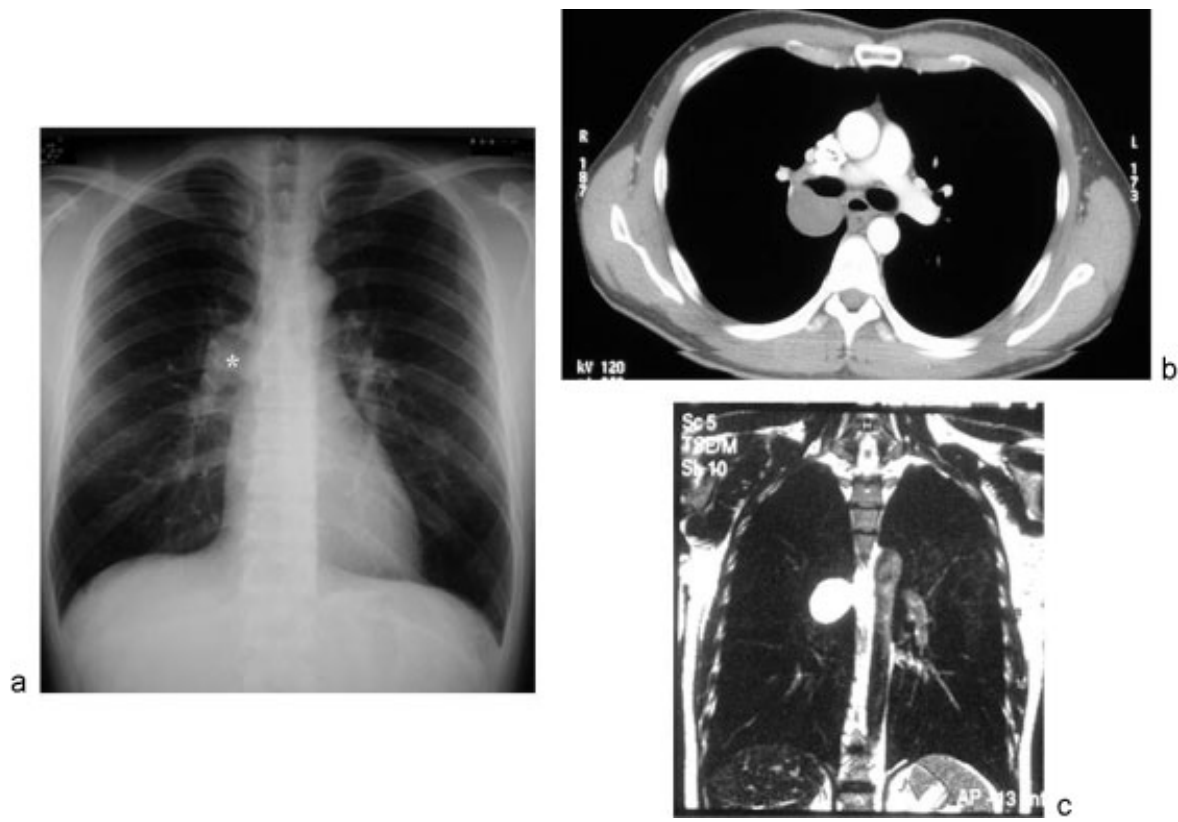

Fig. 1 (a) Chest radiograph showing a smooth, round shadow in the right middle lung field, with an extrapleural sign (*). (b) Enhanced computed tomography scan showing a nonenhanced lesion on the right main bronchus. The lesion was confirmed to be homogeneous, solid, with a clear border. (c) T2-weighted axial magnetic resonance image showing high signal intensity on the right main bronchus.

with a $3.5-\mathrm{cm}$ diameter was located behind the right main bronchus and was resected using ultrasonic coagulating shears. After tumor resection, it was confirmed that the tumor had originated from the pulmonary branch of the right vagus nerve (-Fig. 2a).

The tumor was solid and predominantly yellowish. Microscopically, it comprised cells with long, narrow nuclei as well as wavy bands of spindle-shaped cells with myxomatous interstitial tissue in the background (- Fig. 2b). The histopathological diagnosis was a neurofibroma originating from the pulmonary branch of the right vagus nerve. Immunohistochemistry revealed that the tumor originated from a neuron and stained positive for S-100 and vimentin and negative for actin (data not shown). The patient showed no postoperative complications, and he was discharged two days after surgery.

\section{Conclusion}

Neurogenic tumors are derived from tissue of the neural crest, including cells of the peripheral, autonomic, and paraganglionic nervous systems. About $95 \%$ of posterior mediastinal tumors arise in the intercostal nerve rami or the sympathetic chain region. These tumors are classified on the basis of cell type and comprise approximately 12 to $21 \%$ of all mediastinal tumors, with $95 \%$ occurring in the posterior compartment. ${ }^{4}$ An intrathoracic neurofibroma is rare ${ }^{1,2}$; a comprehensive literature search using PubMed and the Japan Medical Abstracts Society identified 41 cases of neurofibroma, 2,3,5 with 13 of these cases (31.7\%) not accompanied with von Recklinghausen disease. In our case, the tumor originated from the vagus nerve and was located on the right main bronchus. We were unable to find a similar case among the 41 reported cases of intrathoracic neurofibromas.
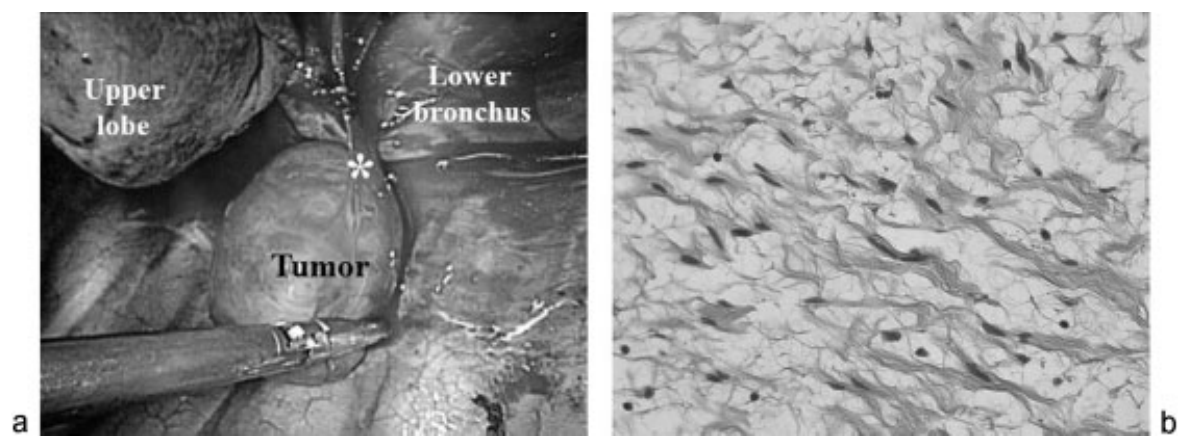

Fig. 2 (a) Intraoperative findings showing a neurogenic tumor originating from the pulmonary branch of the right vagus nerve $\left({ }^{*}\right)$ designates the pulmonary branch of the right vagus nerve. (b) Histological examination showing long, narrow nuclei and wavy bands of spindle-shaped cells with background myxomatous interstitial tissue (hematoxylin and eosin, $400 \times$ magnification). 
Initially, this tumor was diagnosed as a bronchogenic cyst because of its location and MRI findings, similar to a previously reported case in Japan. ${ }^{2}$ We considered clinical followup instead of surgery for this tumor; however, we decided to remove the tumor because it was possible that some of these tumors are malignant; developing in 2 to $16 \%$ patients with neurofibromatosis. ${ }^{3}$ Complete surgical resection is therefore usually recommended for these nerve tumors, accordingly.

\section{Conclusion}

We describe a rare case of an intrathoracic neurofibroma originating from the vagus nerve in a patient without von Recklinghausen disease. Although the diagnosis of neurogenic tumors in the posterior mediastinum is easier than that of tumors in other locations, we performed total resection of the tumor because the possibility of malignancy could not be ruled out.

\section{Acknowledgment}

The authors thank Enago (www.enago.jp) for the English language review.

\section{Conflict of Interest}

Okamoto, Junichi, and other co-authors have no conflict of interest.

\section{References}

1 Saito H, Minamiya Y, Tozawa K, et al. Mediastinal neurofibroma originating from the left intrathoracic phrenic nerve: report of a case. Surg Today 2004;34(11):950-953

2 Hoshino H, Ohbuchi T, Sakon O, et al. [Intrathoracic neurofibroma originating in the left vagus nerve]. (in Japanese) Nihon Kokyuki Gakkai Zasshi 2000;38(1):54-58

3 Kikuchi K, Sakai S, Nemoto E, et al. [Case of neurofibroma originating from the intrathoracic vagal nerve]. (in Japanese) Nihon Kyobu Shikkan Gakkai Zasshi 1983;21(3):288-292

4 Smahi M, Lakranbi M, Ouadnouni $Y$, et al. Intrathoracic phrenic nerve neurofibroma. Ann Thorac Surg 2011;91(4): e57-e58

5 Dabir RR, Piccione W Jr, Kittle CF. Intrathoracic tumors of the vagus nerve. Ann Thorac Surg 1990;50(3):494-497 